\title{
Characteristics of patients with giant cell arteritis who experience visual symptoms
}

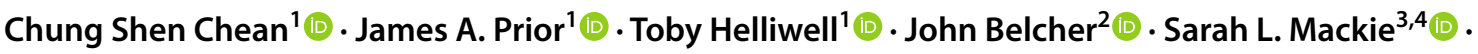

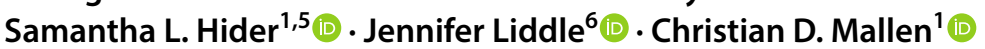

Received: 10 July 2019 / Accepted: 10 August 2019 / Published online: 22 August 2019

(c) The Author(s) 2019

\begin{abstract}
Permanent vision loss is one of the most serious complications of giant cell arteritis (GCA) and therefore prompt diagnosis is paramount. However, diagnosis of GCA remains challenging due to its frequently non-specific presentation. Our aim was to identify differences in the characteristics of GCA patients with, and without, current visual symptoms. A cross-sectional survey was mailed to patients with a GCA Read code entered in their GP electronic medical record. Responders were categorised as those currently reporting a visual symptom or not. We compared general and GCA-specific characteristics in these two groups. The association of diagnostic delay with subsequent experience of visual symptoms was examined using unadjusted and adjusted linear regression analysis. $318 \mathrm{GCA}$ patients responded to the survey $(59.6 \%)$. Responders were predominantly female (69.8\%), with a mean age of 73.7 years (SD 8.2). $28 \%$ reported current visual symptoms. There was no statistically significant difference in the general characteristics between those with and without visual symptoms. Of GCAspecific characteristics, pre-GCA diagnosis of diplopia $(p=0.018)$, temporary $(p \leq 0.001)$ or permanent visual problems $(p=0.001)$ and hoarseness $(p=0.004)$ were more common among those reporting current visual symptoms. There was no association between the extent of diagnostic delay and reporting of current visual symptoms. Though we found few characteristics to distinguish between GCA patients with or without current visual symptoms, diagnostic delay was not associated with current visual symptoms. Our findings highlighted the continued difficulty for clinicians to identify GCA patients at the highest risk of visual complications.
\end{abstract}

Keywords Diagnostic delay · Giant cell arteritis · Visual symptoms

Electronic supplementary material The online version of this article (https://doi.org/10.1007/s00296-019-04422-5) contains supplementary material, which is available to authorized users.

James A. Prior

j.a.prior@keele.ac.uk

Chung Shen Chean

shen920815@gmail.com

Toby Helliwell

t.helliwell@keele.ac.uk

Sarah L. Mackie

s.l.mackie@leeds.ac.uk

Samantha L. Hider

s.hider@keele.ac.uk

Jennifer Liddle

Jennifer.Liddle@newcastle.ac.uk

Christian D. Mallen

c.d.mallen@keele.ac.uk
1 School of Primary, Community and Social Care, Keele University, David Weatherall Building, Keele ST5 5BG, UK

2 Research and Innovation, Wythenshawe Hospital, Manchester University Foundation Hospital Trust, Manchester, UK

3 Leeds Institute of Rheumatic and Musculoskeletal Medicine, University of Leeds, Leeds, UK

$4 \quad$ NIHR Biomedical Research Centre, Leeds Teaching Hospitals NHS Trust, Leeds, UK

5 Haywood Academic Rheumatology Centre, Midlands Partnership Foundation Trust, Stoke-on-Trent, UK

6 Institute of Health and Society, Newcastle University, Newcastle upon Tyne, UK 


\section{Introduction}

Giant cell arteritis (GCA) is the commonest mediumand large-vessel vasculitis of older people, with a peak incidence of 7.4 per 10,000 person years in women aged 70-79 years [1]. This inflammatory condition has a particular predilection for certain arteries, affecting the superficial temporal and posterior ciliary arteries as well as others including the aorta and its proximal branches [2]. Inflammation causes local vascular ischaemia and cytokine release [3], which can result in ischaemic eye manifestations including anterior ischaemic optic neuropathy or retinal artery occlusion. Symptoms experienced may include diplopia, amaurosis fugax and transient or permanent visual loss, one of the most serious consequences of GCA [4, 5]. Early initiation of high-dose corticosteroids (initially 40-60 mg of prednisone equivalent) at onset of symptoms among patients with suspected GCA is the mainstay of treatment, reducing the risk of visual complications, visual loss and other serious outcomes such as stroke or cerebral artery dissection [6].

Despite the importance of patients receiving a prompt diagnosis of GCA in primary care, this remains difficult to achieve. The non-specific nature of many GCA symptoms can lead to patients not consulting, or the general practitioner (GP) associating these symptoms or features, of which many are commonly seen in primary care (e.g. tiredness, fever and weight loss), with conditions other than GCA. GPs may also over-rely on a "classic" GCA presentation (e.g. temporal headache, scalp tenderness and jaw claudication) before considering a GCA diagnosis, although these "classic" symptoms are by no means universal in GCA [7]. A recent clinical review by van der Geest et al. classified GCA as a clinically and immunologically heterogeneous autoimmune disease, dividing it into three subsets: (i) systemic inflammation, (ii) vasculitis of large systemic arteries and (iii) polymyalgia rheumatica [8]. Related to the specific outcome of visual loss, studies have suggested that the risk of visual loss is higher in GCA patients with symptoms such as jaw claudication and in patients who do not have the typical temporal headache [2]. In view of such variability, there is an increasing interest to determine whether clinically important GCA patient subsets, such as those who do develop visual complications, can be identified by distinct patient characteristics.

Improved identification of patients with GCA will reduce persistent diagnostic delay which continues to be a significant problem [7]. A systematic review and meta-analysis identified an average of 8-week diagnostic delay for patients presenting with 'cranial' symptoms and 18 weeks for those with 'non-cranial' symptoms [9]. In view of the severe ophthalmic complications attributed to delayed diagnosis and treatment of GCA, there has been increasing research to reduce these delays. This includes the introduction of fast-track clinics to support the diagnosis of GCA, which have been suggested to be associated with reduced incidence of sight loss [10]. However, such clinics require GPs to be able to accurately identify patients requiring referral and emphasises the need to better understand different GCA patient subsets, especially among GCA patients at higher risk of visual symptoms compared to those without.

The aim of this study was to characterise UK primary care patients with prevalent GCA. Our specific objectives were i) to compare the general and GCA-specific characteristics of those currently reporting visual symptoms and those without visual symptoms and ii) to examine the role of diagnostic delay prior to GCA diagnosis on experience of current visual symptoms.

\section{Materials and methods}

\section{Study design and population}

A self-completion questionnaire was used to collect crosssectional data from patients with GCA. A power calculation determined that the invitation of approximately 500 patients with GCA would provide the minimum 300 patients required to estimate diagnostic delay in this patient group. Adults aged 50 years or older registered to general practices from several regions of England (Clinical Research Network (CRN) areas of: West Midlands (North), Eastern and North Thames) and with any Read coded diagnosis of GCA in the 3 years before the survey were invited to participate in the study. Recruitment occurred from January 2015 to September 2016. Patients identified with GCA by the National Institute for Health Research (NIHR) CRNs were mailed an invitation pack, which included a patient information sheet, survey questionnaire and consent form. This study was given ethical approval by the West Midlands-South Birmingham Local Research Ethics Committee (Ref no: 14/WM/1205) and all patients provided written informed consent.

\section{Data collection}

Data were collected through a self-report postal survey. Socioeconomic data collected included age, gender, body mass index [BMI, from self-report height and weight), level of deprivation (using Indices of Multiple Deprivation (IMD [11])], smoking status and medical comorbidities. Data from a range of GCA-specific characteristics were also collected, including GCA-related symptoms experienced prior to GCA diagnosis, extent of self-reported delay in receiving a GCA diagnosis, age at diagnosis, type of health-care professional 
who diagnosed GCA and doses of prednisolone treatment given when commenced and now currently being taken. Though the questionnaire was not piloted, it was quality assessed internally and included several validated questionnaires, including the Short-Form 12 (SF-12) to measure general physical and mental health [12], the Generalized Anxiety Disorder 7-item (GAD-7) scale [13] and Patient Health Questionnaire (PHQ9) [14] to assess the presence of anxiety and depression, respectively, and finally the Visual Function Questionnaire-14 (VF-14) as a method of assessing the quality of visual function [15].

\section{Statistical analysis}

Survey responders were stratified into two groups: (i) GCA patients with current visual symptoms and (ii) GCA patients without current visual symptoms. The visual symptoms group was defined as those currently reporting having at least one of three visual symptoms attributed to their GCA: double vision, temporary vision problems and permanent vision problems.

The general and GCA-specific characteristics of the study sample were initially summarised using descriptive statistics. The mean age [with standard deviation (SD)] and gender ratio were reported, BMI was categorised into three groups ( $<24.9 \mathrm{~kg} / \mathrm{m}^{2}$ healthy weight, $25.0-29.9 \mathrm{~kg} /$ $\mathrm{m}^{2}$ overweight, $>30.0 \mathrm{~kg} / \mathrm{m}^{2}$ obese), as was IMD (the $20 \%$ least deprived, mid-deprived and $20 \%$ most deprived). Smoking status classified responders into those who had never smoked, previously smoked or were current smokers. Responders reported whether they had previously received a diagnosis for one of the listed comorbidities (hypertension, hyperlipidaemia, polymyalgia rheumatica, diabetes, stroke, cancer, cataracts and glaucoma). The presence of anxiety and depression was determined by a score $>10$ on the GAD7 or PHQ-9 measures, respectively. The VF-14 is scored on a scale of 0 (not capable) to 100 (perfectly capable) related to aspects of daily living affected by vision.

With regard to GCA-specific characteristics, mean age at diagnosis was reported and using this, disease duration was calculated [median, interquartile range (IQR)]. The prevalence of any of 18 potential GCA symptoms could be reported by survey responders (headache/head pain, tiredness/fatigue, temporary vision problems, scalp tenderness, shoulder pain or stiffness, pain or difficulty chewing, ear pain, loss of appetite, pain in lower body when walking, double vision, high temperature, hip pain or stiffness, toothache, hoarseness, weight loss, pain in upper body when walking, dry cough and permanent vision problems) and the patients' historical and current prednisolone use were reported as absolute values and percentages. Use of prednisolone was further described by asking patients to record the starting dose and current daily doses. Both were categorised into three groups, starting doses by $<40 \mathrm{mg}, 40-60 \mathrm{mg}$ or $>60 \mathrm{mg}$ and current prednisolone daily dose by those taking $<7.5 \mathrm{mg}, 7.5-10 \mathrm{mg}$, or $>10 \mathrm{mg}$. Three aspects of delay were measured: (i) the time taken for the patient to consult their doctor (consultation delay), (ii) time between first consultation and GCA diagnosis (diagnostic delay) and (iii) the total time between symptom onset and GCA diagnosis (total delay). Each delay category was reported as the median (IQR) number of days of delay.

Comparisons of data between GCA patients with and without current visual symptoms were made using $t$ test, Mann-Whitney $U$ test or Fisher exact test as appropriate. Given that delay data were skewed, multivariable linear regression analysis with bootstrapping was used to examine the association between the number of days of delay for each delay category experienced (consultation, diagnostic and total) and the presence of visual symptoms among GCA patients, as compared to GCA patients without visual symptoms. We constructed bias-corrected and accelerated (BCa) bootstrapped $95 \%$ confidence intervals for the regression coefficients as these are robust to non-normality [16]. The analysis was initially unadjusted, followed by adjustment for age, gender and deprivation. Age, as a continuous predictor, had a linear relationship with the delay interval, and not a non-linear effect such as age squared.

\section{Results}

Of the 534 patients with GCA who were mailed a questionnaire, 318 responded (adjusted response rate of 59.6\%) from a total of 130 general practices. The mean age (SD) of responders was 73.7 (8.2) years, 222 patients (69.8\%) were female, 79 patients $(24.7 \%)$ were obese and 151 patients (47.6\%) had never smoked. Of responders, 90 (28.3\%) reported a current visual symptom, with just over half (55.0\%) of these being new cases who reported visual symptoms developing only after diagnosis. 228 survey responders reported no current visual symptoms at the time of the survey $(71.7 \%)$.

\section{General characteristics}

There was no significant difference between the demographic factors (age, gender, deprivation status, BMI and smoking status) of GCA patients with or without current visual symptoms. Of the self-report comorbidities, anxiety $(p=0.023)$ was more frequent in those with current visual symptoms (Table 1). GCA patients without current visual symptoms had higher (better) mean SF-12 PCS score of 40.9 (SD 12.3, $p=0.0001$ ) and mean SF-12 MCS score of 47.1 (SD 11.1, $p=0.028$ ) as compared to patients with current GCA visual symptoms. GCA patients without visual 
Table 1 Baseline characteristics of patients with GCA who are currently experiencing visual symptoms and those without current visual symptoms

\begin{tabular}{|c|c|c|c|}
\hline & $\begin{array}{l}\text { GCA with current visual } \\
\text { symptoms }(N=90)\end{array}$ & $\begin{array}{l}\text { GCA without current visual } \\
\text { symptoms }(N=228)\end{array}$ & $p$ value \\
\hline Mean age (SD) & $72.6(8.4)$ & $74.1(8.0)$ & 0.131 \\
\hline \multicolumn{4}{|l|}{ Gender $(n, \%)$} \\
\hline Male & $26(28.9)$ & $70(30.7)$ & \multirow[t]{2}{*}{0.788} \\
\hline Female & $64(71.1)$ & $158(69.3)$ & \\
\hline \multicolumn{4}{|l|}{ Deprivation status $(n, \%)$} \\
\hline $20 \%$ least deprived & $17(18.9)$ & $46(20.4)$ & \multirow[t]{3}{*}{0.895} \\
\hline $60 \%$ middle & $56(62.2)$ & $133(59.2)$ & \\
\hline $20 \%$ most deprived & 17 (18.9) & $46(20.4)$ & \\
\hline \multicolumn{4}{|l|}{$\operatorname{BMI}(n, \%)$} \\
\hline Healthy weight $\left(<24.9 \mathrm{~kg} / \mathrm{m}^{2}\right)$ & $27(32.9)$ & $93(43.5)$ & \multirow[t]{3}{*}{0.146} \\
\hline Overweight $\left(25.0-29.9 \mathrm{~kg} / \mathrm{m}^{2}\right)$ & $29(35.4)$ & $74(34.6)$ & \\
\hline Obese $\left(>30.0 \mathrm{~kg} / \mathrm{m}^{2}\right)$ & $26(31.7)$ & $47(21.9)$ & \\
\hline \multicolumn{4}{|l|}{ Smoking status $(n, \%)$} \\
\hline Never smoked & $42(47.2)$ & $108(47.8)$ & \multirow[t]{3}{*}{0.796} \\
\hline Previous smoker & $40(44.9)$ & $105(46.4)$ & \\
\hline Current smoker & $7(7.9)$ & $13(5.8)$ & \\
\hline \multicolumn{4}{|l|}{ Comorbidities at baseline $(n, \%)$} \\
\hline Hypertension & $53(58.9)$ & $111(48.9)$ & 0.135 \\
\hline Hyperlipidaemia & $35(38.9)$ & $93(41.0)$ & 0.800 \\
\hline Polymyalgia rheumatic & $26(28.9)$ & $71(31.3)$ & 0.787 \\
\hline Depression & $25(32.5)$ & $49(24.6)$ & 0.187 \\
\hline Anxiety & $24(30.8)$ & $38(18.3)$ & $0.023 *$ \\
\hline Diabetes & $23(25.6)$ & $36(15.9)$ & 0.055 \\
\hline Stroke & $9(10.0)$ & $19(8.4)$ & 0.666 \\
\hline Cancer & $7(7.8)$ & $25(11.0)$ & 0.535 \\
\hline Cataracts & $29(32.2)$ & $70(30.8)$ & 0.893 \\
\hline Glaucoma & $8(8.9)$ & $18(7.9)$ & 0.821 \\
\hline
\end{tabular}

*Significance and $p \leq 0.05$ symptoms also had a higher (better visual function) mean VF-14 score (Mean 86.9, SD 17.6) as compared to the other group (Mean 71.9, SD 25.9) and this was statistically significant $(p=0.000)$ (Table 2$)$.

\section{GCA-specific characteristics}

There was no difference in the age at which GCA patients were diagnosed or the disease duration for those with or without current visual symptoms. The three commonest symptoms experienced by GCA patients with current visual symptoms prior to their diagnosis were headache $(82.2 \%)$, tiredness/fatigue $(63.3 \%)$ and temporary vision problems (52.2\%), whereas among GCA patients without current visual symptoms, the commonest symptoms were headache $(87.3 \%)$, scalp tenderness $(59.7 \%)$ and tiredness/fatigue $(57.8 \%)$. Comparing groups of GCA patients with visual symptoms to those without, the proportions of patients with hoarseness, double vision, temporary visual problems and permanent visual problems were significantly higher for those with current visual symptoms. Comparing GCA patients with current visual symptoms to those without, a higher proportion of patients in the former group $(25.0 \%)$ than the latter (12.7\%) had GCA first diagnosed by an ophthalmologist $(p=0.010)$ (Table 2).

\section{Delays in receiving GCA diagnosis}

Of the total survey responders, the median number of days of delay in seeking health care (consultation delay) was 14 (IQR 7, 42), diagnostic delay was 14 (IQR 4, 35) and total days of delay was 35 (IQR 18, 91). Using linear regression analyses with bootstrapping, adjusted for age, gender and deprivation, we found no significant difference between the number of days of consultation $[-1.19(-29.85,27.46)]$, diagnostic $[-6.07(-28.23,16.10)]$ or total delay $[-2.29$ ( $-50.97 .46 .40)]$ experienced by GCA patients with a current visual symptom, compared to those with no current visual symptom (Table 3 ). 
Table 2 GCA-specific characteristics of patients with and without visual symptoms

\begin{tabular}{|c|c|c|c|}
\hline & $\begin{array}{l}\text { GCA with current visual } \\
\text { symptoms }(N=90)\end{array}$ & $\begin{array}{l}\text { GCA without current } \\
\text { visual symptoms }(N=228)\end{array}$ & $p$ value \\
\hline Age at diagnosis (mean; SD) & $69.6(8.5)$ & $71.5(8.1)$ & 0.072 \\
\hline Disease duration (median years; IQR) & $2.7(1.1,3.2)$ & $2.1(1.2,3.1)$ & 0.815 \\
\hline \multicolumn{4}{|l|}{ Symptoms before GCA diagnosis $(n, \%)$} \\
\hline Headache/head pain & $74(82.2)$ & $199(87.3)$ & 0.284 \\
\hline Tiredness/fatigue & $57(63.3)$ & $132(57.8)$ & 0.447 \\
\hline Temporary vision problems & $47(52.2)$ & $70(30.7)$ & $0.000^{*}$ \\
\hline Scalp tenderness & $44(48.9)$ & $136(59.7)$ & 0.102 \\
\hline Shoulder pain or stiffness & $41(45.6)$ & $105(46.1)$ & 1.000 \\
\hline Pain or difficulty chewing & $39(43.3)$ & $104(45.6)$ & 0.803 \\
\hline Ear pain & $30(33.3)$ & $53(23.3)$ & 0.088 \\
\hline Loss of appetite & $28(31.1)$ & $54(23.7)$ & 0.200 \\
\hline Pain in lower body when walking & $27(30.0)$ & $65(28.5)$ & 0.785 \\
\hline Double vision & $22(24.4)$ & $30(13.2)$ & $0.018^{*}$ \\
\hline High temperature & $21(23.3)$ & $32(14.0)$ & 0.065 \\
\hline Hip pain or stiffness & $19(21.1)$ & $58(25.4)$ & 0.469 \\
\hline Toothache & $18(20.0)$ & $41(18.0)$ & 0.749 \\
\hline Hoarseness & $17(18.9)$ & $16(7.0)$ & $0.004 *$ \\
\hline Weight loss & $16(17.8)$ & $48(21.1)$ & 0.642 \\
\hline Pain in upper body when walking & $15(16.7)$ & $30(13.2)$ & 0.475 \\
\hline Dry cough & $13(14.4)$ & $34(14.9)$ & 1.000 \\
\hline Permanent vision problems & $11(12.2)$ & $6(2.6)$ & $0.001 *$ \\
\hline \multicolumn{4}{|l|}{ Who diagnosed GCA $(n, \%)$} \\
\hline GP & $56(63.6)$ & $163(71.5)$ & 0.177 \\
\hline Rheumatologist & $38(43.2)$ & $91(39.9)$ & 0.611 \\
\hline Ophthalmologists & $22(25.0)$ & $29(12.7)$ & $0.010^{*}$ \\
\hline Geriatrician & $1(1.1)$ & $2(0.8)$ & 1.000 \\
\hline Others & $15(17.1)$ & $34(14.9)$ & 0.729 \\
\hline \multicolumn{4}{|l|}{ Prednisolone use } \\
\hline Ever taken prednisolone? & $87(97.8)$ & $217(95.6)$ & 0.520 \\
\hline Still taking prednisolone? & $64(71.9)$ & $148(68.5)$ & 0.587 \\
\hline \multicolumn{4}{|l|}{ Current prednisolone daily dose } \\
\hline$<7.5 \mathrm{mg}$ & $37(62.7)$ & $90(65.2)$ & 0.260 \\
\hline On maintenance $7.5-10 \mathrm{mg}$ & $7(11.9)$ & $25(18.1)$ & \\
\hline$>10 \mathrm{mg}$ & $15(25.4)$ & $23(16.7)$ & \\
\hline \multicolumn{4}{|l|}{ Prednisolone starting dose } \\
\hline$<40 \mathrm{mg}$ & $21(26.9)$ & $48(24.6)$ & 0.488 \\
\hline $40-60 \mathrm{mg}$ & $51(65.4)$ & $138(70.8)$ & \\
\hline$>60 \mathrm{mg}$ & $6(7.7)$ & $9(4.6)$ & \\
\hline VF-14 (mean; SD) & $71.9(25.9)$ & $86.9(17.6)$ & $0.000^{*}$ \\
\hline SF-12 PCS score (mean; SD) & $34.6(10.8)$ & $40.9(12.3)$ & $0.0001 *$ \\
\hline SF-12 MCS score (mean; SD) & $43.8(11.4)$ & $47.1(11.1)$ & $0.028^{*}$ \\
\hline
\end{tabular}

*Significance and $p \leq 0.05$

\section{Discussion}

Our cross-sectional analysis of patients with prevalent GCA from primary care has identified that approximately a quarter of our sample were currently experiencing some form of visual symptom, and of these, approximately half had had no visual symptoms before their diagnosis of GCA. There were few significant differences between patients reporting current visual symptoms and those without. As would be expected, patients with current visual symptoms reported more visual symptoms prior to GCA diagnosis, were more likely to have had their GCA 
Table 3 Association between GCA patients with or without visual symptoms and the difference in the number of days of delay experienced in receiving their diagnosis of GCA

Median days of delay (IQR)

\begin{tabular}{ll} 
Linear regression analysis & \\
\hline $\begin{array}{l}\text { Unadjusted mean difference in days of } \\
\text { delay }(95 \% \mathrm{CI})\end{array}$ & $\begin{array}{l}\text { Adjusted mean difference } \\
\text { in days of delay }(95 \% \\
\end{array}$ \\
CI)*
\end{tabular}

Consultation delay

No visual symptoms

Visual symptoms

Diagnostic delay

No visual symptoms

Visual symptoms

Total delay

No visual symptoms

Visual symptoms
$14.0(7.0,58.4)$

$14.0(5.0,30.4)$

$14.0(4.0,42.0)$

$14.0(4.0,30.4)$

$36.2(19.0,93.0)$

$35.0(16.0,65.8)$
Ref

-1.37 (-26.2, 23.46)

Ref

$-7.13(-30.33,16.08)$

Ref

$-3.37(-49.47,42.72)$
Ref

$-1.19(-29.85,27.46)$

Ref

$-6.07(-28.23,16.10)$

Ref

$-2.29(-50.97,46.40)$

*Adjusted by age, gender and deprivation

diagnosed by an ophthalmologist and reported poorer visual function at the time of the survey. Importantly, GCA patients with current visual symptoms had not taken any longer to consult their GP and did not experience a greater delay in receiving a diagnosis as compared to those without visual symptoms. However, despite similar starting and current prednisolone doses between the two groups, GCA patients with current visual symptoms reported significantly more anxiety and poorer general physical and mental health. Finally, of the many non-vision-related symptoms reported prior to GCA diagnosis, only hoarseness was more common in GCA patients with current visual symptoms.

We found the prevalence of current visual symptoms in our sample to be similar to several other GCA studies. Population-based cohort studies performed by Singh et al. in the USA and Salvarani et al. in Italy reported $23 \%$ and $30.1 \%$ of patients with visual changes, respectively $[17,18]$. Another prospective study of 174 patients showed the occurrence of visual ischaemic manifestations among $28 \%$ of patients, with permanent visual loss in $13 \%$ of them [19]. In patients with biopsy-proven GCA, Gonzalez-Gay et al. performed a retrospective study at a single reference hospital in Spain, which showed that $26.1 \%$ had visual ischaemic complications and $14.9 \%$ had irreversible blindness [20]. However, lower rates of visual complications have also been reported $(12-15 \%)[21,22]$. Any discrepancy with our findings could be explained by the differences in the study design and in the case mix of included patients. Our reported proportion of visual symptoms could also be an underestimation, as samples from these previous studies were identified through hospital episodes and also visual complications were not well defined in many studies, causing difficulties in accurate case ascertainment.
Our study showed that GCA patients with current visual symptoms experienced significantly more anxiety and poorer general physical and mental health at baseline, assessed by SF-12 summary scores. Though a qualitative study performed in the UK also found that anxiety about future visual loss was an important concern of GCA patients [23], this is in contrast to other studies. Jobard et al. and Kupersmith et al. found that GCA visual complications did not have any major impact on health-related quality of life (QoL), as assessed using the SF-36 score [24, 25]. However, neither of the aforementioned studies distinguished between patients with and without visual symptoms.

As we appreciate that vision ranks as the number one concern among patients with GCA [24], our study used the VF-14 [26] as a measure of visual function among GCA subjects. Expectedly, GCA patients with current visual symptoms had lower (worse) VF-14 score than those without visual symptoms. However, as context for the visual "situation" of our current visual symptom group, Valderas et al. found that for a patient with a VF-14 of 71 (comparable to our group), this would correspond to a visual function allowing the patient to be 'doing fine handwork', 'watching TV' or 'recognising people' without difficulty, but with some difficulty 'reading small print' and unable to 'drive at night' [27]. However, as the VF-14 has only been validated in ophthalmological conditions such as cataract, glaucoma, corneal transplants, keratoconus and dry eye disease, such comparisons should be interpreted with caution [26].

The multitude of symptoms experienced prior to GCA diagnosis in our sample was extensive. However, the only non-vision-related symptom to be reported prior to GCA diagnosis and to distinguish those patients with or without current visual symptoms was hoarseness. Hoarseness has only previously been reported infrequently in case reports 
or case series $[28,29]$. Our findings are in contrast to previous research which showed that patients with visual symptoms were less likely to have headaches, fever and a palpable tender temporal artery (not recorded by our study), and more likely to have jaw claudication [17, 30]. Many previous studies have also found an inverse relationship between fever or high temperature and the development of ischaemic complications [31], and that lower inflammatory responses occurred in GCA patients with visual symptoms as compared to those without visual symptoms [19]. This could be explained by differences in consultation patterns, in that those with fever or significant systemic symptoms may be more likely to consult or receive glucocorticoid treatment and hence be at lower risk of visual complications.

Due to the non-specific symptoms of GCA, diagnostic delay is common [9], but previous research has shown conflicting evidence of association of diagnostic delays and visual loss. Ezeonyeji et al. highlighted that diagnostic delay is associated with increased risk of irreversible visual loss among patients with GCA [7], but GonzalezGay et al. showed no association of the length of delay of GCA diagnosis with the risks of visual manifestations and permanent visual loss [20]. However, delay is multifaceted and our work has examined both delays which are predominately related to the actions of patients (consultation delay) and to those actions of the clinician (diagnostic delay). We found no significant difference in reported consultation or diagnostic delay between GCA patients with current visual symptoms versus those without visual symptoms.

\section{Strengths and limitations}

The strength of our study is the provision of new information on general and GCA-specific characteristics of an important subset of patients with GCA for which large datasets are limited. This study, which recruited patients from several regions of England, recorded extensive information regarding symptoms, both before GCA diagnosis and several years after. This also separates out consultation and diagnostic delay.

Limitations of our work include the use of a cross-sectional, self-report mailed survey, as such a design may have introduced both selection and recall bias. Regarding selection bias, recruitment directly through the CRNs means we were unable to determine which patients were unwilling to take part and whether these are a different subset of patients with GCA, for example, such patients could have included those with poor vision and therefore have been limited in their ability to complete a postal questionnaire or simply unwilling. Regarding recall bias, patients may have had problems answering questions accurately which related to previous events several years prior, i.e. symptoms at the point of a diagnosis. However, this design was the most practical for the collection of detailed data from elderly patients (or their proxies) across several different geographical areas, and delay and symptom prevalence data reflect previous research, providing confidence in the generalisability of our results. A further limitation relates to the cause of symptoms. Though our questionnaire requested the reporting of symptoms in the context of "being caused by your GCA", there is a potential that these were caused by other comorbidities or even a consequence of GCA treatment; oral glucocorticoid treatment can also affect vision via several mechanisms including accelerated cataract formation and changes in blood glucose levels [32]. Therefore, this selfreporting of symptoms could have been due to heterogeneous aetiologies and there is the potential for misclassification of outcome. Finally, due to the rarity of GCA and therefore a smaller sample, we were required to group several visual symptoms together for analysis and were unable to stratify the 'current visual symptoms' group further into those with symptoms before or after GCA diagnosis. Despite our inability to tease out these differences, our work remains a much needed comparison of the visual symptoms subgroup of GCA patients.

In conclusion, visual symptoms remain a serious and common problem among patients with GCA, both before and several years after diagnosis. Early, appropriate treatment is important as GCA-related symptoms are preventable and if GCA-related visual symptoms are present, then urgent treatment and referral to ophthalmology is essential to prevent progression and ultimately complete blindness. However, we found little to distinguish this subset of GCA patients from those who did not report current visual symptoms, highlighting the continued difficulty for health-care professionals (and GPs in particular) to identify these patients promptly, even those with serious symptoms. Though both patient and health-care-related delay persists, this appears to be less than previously reported and visual symptoms may not be related to the extent of the delay experienced. Longitudinal analysis on this topic is needed to fully characterise GCA patients prior to or at disease onset, to reduce diagnostic and subsequently treatment delays.

Author contributions JAP, JL and CDM made substantial contributions to the acquisition of funding and conception and design of the work. CSC, JP, TH, SLM, SLH, JB and CDM collected, analysed or interpreted data. CSC drafted the first version of the manuscript, with all authors supporting subsequent iterations, approving the submitted version and taking full responsibility for the integrity of the study and the final version of the manuscript.

Funding Study funding was secured by JAP from the National Institute for Health Research (NIHR) School for Primary Care Research (SPCR), Grant Reference Number: 294. CDM was funded by the NIHR Collaborations for Leadership in Applied Health Research and Care (CLAHRC), West Midlands, the NIHR SPCR and an NIHR Research 
Professorship in General Practice (NIHR-RP-2014-04-026). TH was funded by an NIHR Clinical Lectureship. SM was funded by an NIHR Clinician Scientist Fellowship award. The study sponsors had no role in study design; in the collection, analysis and interpretation of data; in the writing of the report; and in the decision to submit the paper for publication. The views and opinions expressed therein are those of the authors and do not necessarily reflect those of the NIHR (UK). The views expressed are those of the author(s) and not necessarily those of the NHS, the NIHR or the Department of Health and Social Care.

\section{Compliance with ethical standards}

Conflict of interest The authors declare that they have no conflicts of interest.

Open Access This article is distributed under the terms of the Creative Commons Attribution 4.0 International License (http://creativeco mmons.org/licenses/by/4.0/), which permits unrestricted use, distribution, and reproduction in any medium, provided you give appropriate credit to the original author(s) and the source, provide a link to the Creative Commons license, and indicate if changes were made.

\section{References}

1. Petri H, Nevitt A, Sarsour K et al (2015) Incidence of giant cell arteritis and characteristics of patients: data-driven analysis of comorbidities. Arthritis Care Res (Hoboken) 67(3):390-395

2. Vodopivec I, Rizzo JF III (2018) Ophthalmic manifestations of giant cell arteritis. Rheumatology 57(suppl_2):ii72

3. Barraclough K, Mallen CD, Helliwell T et al (2012) Diagnosis and management of giant cell arteritis. Br J Gen Pract 62(599):329-330

4. Figus M, Talarico R, D'Ascanio A et al (2013) Ocular involvement in giant cell arteritis. Clin Exp Rheumatol 31(1 Suppl 75):S96

5. Hayreh SS, Podhajsky PA, Zimmerman B (1998) Occult giant cell arteritis: ocular manifestations. Am J Ophthalmol 125(4):521-526

6. Dasgupta B, Borg FA, Hassan N et al (2010) BSR and BHPR guidelines for the management of giant cell arteritis. Rheumatology 49(8):1594-1597

7. Ezeonyeji AN, Borg FA, Dasgupta B (2011) Delays in recognition and management of giant cell arteritis: results from a retrospective audit. Clin Rheumatol 30(2):259-262

8. van der Geest Kornelis SM, Sandovici M, van Sleen Y et al (2018) What is the current evidence for disease subsets in giant cell arteritis? Arthritis Rheumatol 70(9):1366-1376

9. Prior JA, Ranjbar H, Belcher J et al (2017) Diagnostic delay for giant cell arteritis-a systematic review and meta-analysis. BMC Med 15(1):120

10. Patil P, Williams M, Maw WW et al (2015) Fast track pathway reduces sight loss in giant cell arteritis: results of a longitudinal observational cohort study. Clin Exp Rheumatol 33(2 Suppl 89):6

11. Communities and neighbourhoods (2015) The English indices of deprivation 2015. https://www.gov.uk/government/statistics/engli sh-indices-of-deprivation-2015. Accessed 15 Feb 2019

12. Ware J Jr, Kosinski M, Keller SD (1996) A 12-item short-form health survey: construction of scales and preliminary tests of reliability and validity. Med Care 34(3):220-233

13. Spitzer RL, Kroenke K, Williams J et al (2006) A brief measure for assessing generalized anxiety disorder: the GAD-7. Arch Intern Med 166:1092-1097

14. Kroenke K, Spitzer RL, Williams JB (2001) The PHQ-9: validity of a brief depression severity measure. J Gen Intern Med 16:606-613
15. Kishimoto F, Ohtsuki H (2012) Comparison of VF-14 scores among different ophthalmic surgical interventions. Acta Med Okayama 66(2): 101-110

16. Efron B, Tibshirani RJ (1994) An introduction to the bootstrap. CRC Press, London

17. Singh AG, Kermani TA, Crowson CS et al (2015) Visual manifestations in giant cell arteritis: trend over 5 decades in a populationbased cohort. J Rheumatol 42(2):309-315

18. Salvarani C, Cimino L, Macchioni P et al (2005) Risk factors for visual loss in an Italian population-based cohort of patients with giant cell arteritis. Arthritis Rheum 53(2):293-297

19. Liozon E, Herrmann F, Ly K et al (2001) Risk factors for visual loss in giant cell (temporal) arteritis: a prospective study of 174 patients. Am J Med 111(3):211-217

20. González-Gay MA, García-Porrúa C, Llorca J et al (2000) Visual manifestations of giant cell arteritis. Trends and clinical spectrum in 161 patients. Medicine 79(5):283-292

21. Bengtsson BA, Malmvall BE (1981) The epidemiology of giant cell arteritis including temporal arteritis and polymyalgia rheumatica. Incidences of different clinical presentations and eye complications. Arthritis Rheum 24(7):899-904

22. Ji J, Dimitrijevic I, Sundquist J et al (2017) Risk of ocular manifestations in patients with giant cell arteritis: a nationwide study in Sweden. Scand J Rheumatol 46(6):484-489

23. Liddle J, Bartlam R, Mallen CD et al (2017) What is the impact of giant cell arteritis on patients' lives? A UK qualitative study. BMJ Open 7(8):e017073

24. Jobard S, Magnant J, Blasco H et al (2017) Quality of life of patients treated for giant cell arteritis: a case-control study. Clin Rheumatol 36(9):2055-2062

25. Kupersmith MJ, Speira R, Langer R et al (2001) Visual function and quality of life among patients with giant cell (temporal) arteritis. J Neuroophthalmol 21(4):266-273

26. Steinberg EP, Tielsch JM, Schein OD et al (1994) The VF-14: an index of functional impairment in patients with cataract. Arch Ophthalmol 112(5):630-638

27. Valderas JM, Alonso J, Prieto L et al (2004) Content-based interpretation aids for health-related quality of life measures in clinical practice. An example for the visual function index (VF-14). Qual Life Res 13(1):35-44

28. Larson TS, Hall S, Hepper N et al (1984) Respiratory tract symptoms as a clue to giant cell arteritis. Ann Intern Med 101(5):594-597

29. Ali MN (2005) Hoarse voice and visual loss. Br J Ophthalmol 89(2): 240

30. Saleh M, Turesson C, Englund M et al (2016) Visual complications in patients with biopsy-proven giant cell arteritis: a population-based study. J Rheumatol 43(8):1559-1565

31. Berger CT, Wolbers M, Meyer P et al (2009) High incidence of severe ischaemic complications in patients with giant cell arteritis irrespective of platelet count and size, and platelet inhibition. Rheumatology 48(3):258-261

32. Chirikov VV, Shah R, Kwon Y et al (2019) Oral corticosteroid exposure and increased risk of related complications in patients with noninfectious intermediate, posterior, or panuveitis: realworld data analysis. Ophthalmic Epidemiol 26:27-46. https:// doi.org/10.1080/09286586.2018.1513042

Publisher's Note Springer Nature remains neutral with regard to jurisdictional claims in published maps and institutional affiliations. 